\title{
Evaluating the Feasibility of a Socio-Spiritual Intervention To Improve Quality of Life of Adults Living With Cancer and Their Family Caregivers: A Randomised Controlled Trial.
}

Israel Gabriel ( $\nabla$ israelchristson@gmail.com )

Griffith University https://orcid.org/0000-0002-5663-450X

Debra Creedy

Griffith University

Elisabeth Coyne

Griffith University

\section{Study Protocol}

Keywords: adult patient, cancer, family caregiver, health literacy, informal caregiver, quality of life, Nigeria, socio-spiritual intervention.

Posted Date: May 6th, 2021

DOl: https://doi.org/10.21203/rs.3.rs-476537/v1

License: (c) (1) This work is licensed under a Creative Commons Attribution 4.0 International License.

Read Full License 


\section{Abstract}

Background: Despite high psychosocial needs that negatively affect the quality of life of adults living with cancer and their family caregivers, few interventions are culturally sensitive for people in low-income countries. This protocol describes a randomised controlled trial that aims to test the feasibility and efficacy of a socio-spiritual intervention to improve spiritual, family/social and information needs; health literacy; and quality of life of adult Nigerians living with cancer and their family caregivers.

Methods: This two-arm randomised controlled trial will recruit 152 adults living with cancer and their family caregivers (76 dyads). Participants will be recruited from a publicly funded hospital outpatient clinic in Nigeria. Eligible participants will be randomly assigned to either the intervention or control group at a 1:1 ratio. The intervention consists of four weekly face-to-face sessions with a focus on psychosocial needs, communication and building trusting relationships, and understanding strengths and resources of the family to build spiritual support. Control participants will receive usual care. The primary outcome is feasibility (rates of eligibility, acceptance, retention, assessment process, and fidelity) of the intervention. Secondary outcomes are quality of life (measured by the Functional Assessment of Chronic Illness Therapy-Spirituality [FACIT-Sp]), spiritual needs (Spiritual Needs Assessment for Patients [SNAP]), social support (Multidimensional Scale of Perceived Social Support [MSPSS]), and information needs (Subscale of the Comprehensive Needs Assessment Tool for Cancer).

Discussion: Nigeria has the highest rate of extreme poverty globally, with increasing rates of cancer mortality. Testing the feasibility of social-spiritual interventions in resource-poor settings is essential to establish efficacy, sustainability, and support coping strategies of individuals with cancer and their caregivers. Demonstrated feasibility will enhance implementation of such interventions in clinical practice and communities in other low and middle-income countries.

Trial registration: The trial was registered with the Pan African Clinical Trial Registry (www.pactr.org, identifier: PACTR202007829295775) on $15^{\text {th }}$ June 2020.

\section{Background}

Cancer mortality is on the rise in sub-Saharan Africa, where there is limited health care infrastructure (1). According to cancer incidence estimates, about 238,000 new cancer cases in this region will occur annually, with an almost triple rise in the number of new cancer cases expected by 2050 (2). Several subSaharan African countries are under-resourced to provide treatment for adults with cancer despite significant progress in cancer management globally (3). Lack of resources place many adults with cancer in the care of close relatives who may have little or no capability (4). While adults with cancer struggle with symptoms and uncertainty, family caregivers may also suffer significant long-term psychosocial burden $(5,6)$. Psychosocial burden may be associated with a range of needs related to information, family/social, spiritual, practical, psychological, and physical support (7). 
Cancer health literacy is critical for individuals living with cancer and their family caregivers in subSaharan Africa, where socio-cultural beliefs influence help-seeking and care behaviours (8). Socio-cultural beliefs about cancer in sub-Saharan Africa contribute to late presentation, failure to adhere to treatment and discontinuation of therapy (9). Although the provision of relevant education about the disease to both adults with cancer and family caregivers may contribute to better decision-making, socio-cultural beliefs must also be considered. In traditional African culture, diseases such as cancer are conceptualised as a connection with the supernatural or ancestral forces. This understanding influences how the disease is perceived, and steps taken towards diagnosis and management (10).

Family/social need is another foremost contributor of quality of life (QoL) and plays an essential role in cancer care. It encompasses practical help, personal care for patients, preservation or enhancement of social networks, and emotional support (11). The ability of the family to maintain cohesion and adequate support for each other is an essential consideration in cancer care. A study of psychosocial needs and health-related QoL among 600 colorectal cancer survivors in the United Kingdom (U.K) found that participants with one or more unmet needs had decreased QoL (12). Similarly, the prevalence and impact of psychosocial needs were predictive of QoL among 52 South Korean women with recurrent breast cancer (13). Psychosocial needs are amenable to intervention. This is consistent with systematic reviews and meta-analyses on the positive effects of psychosocial interventions on QoL in cancer patients (14, 15). Additionally, various family-based interventions have been used to support people living with cancer, and their families manage the condition and caregiving burden and improve QoL (16-18). But, these studies were mainly conducted in high-income countries with no published studies emerging from Africa, or Nigeria in particular (19). Interventions tested in high-income countries may not apply in low-middleincome countries (LMICs) as content may lack specific components that are important to African people, such as spirituality, concept of familism, and contextualised information about cancer.

A recent needs survey among 240 adult Nigerians living with cancer and their family caregivers and found a reduction in QoL (20). Information (90.8\%) and spiritual support (85\%) were the domains of highest need among adults with cancer, while family/social support (85\%) and spiritual support (81.7\%) ranked the highest among family caregivers. Needs (spiritual, social, and information) were negatively related to QoL. (20).

\section{Conceptual framework}

The proposed study is guided by a spiritual framework adapted from Hodge (21). The framework identifies seven discrete explanatory mechanisms, or pathways through which spirituality produces beneficial outcomes: health promoting behaviours and lifestyles, social support, psychodynamics of ritual, psychodynamics of cognitive schemata, ego challenges, quantum effects, and supernatural effects. For this study, five specific issues were identified within the pathways (health promoting behaviours and lifestyles, social support, psychodynamics of ritual, psychodynamics of cognitive schemata, and supernatural effects) to explain how spirituality influences QoL (see Figure 1). This framework provides a theoretical approach to assessing change in QoL. 


\section{Rationale and previous work}

This study includes four work package of the socio-spiritual intervention for adult Nigerians with cancer and their family caregivers. Work Package 1 included a systematic review (19), while Work Package 2 included a quantitative needs survey of 240 adult Nigerians living with cancer and their family caregivers (20). Based on the data obtained in work packages one and two, the Behaviour Change Wheel (BCW) (22) was used to develop the intervention (work package three). Target behaviours were described as (1) involvement in socio-spiritual behaviours to increase QoL across the study population, and (2) appropriate knowledge of the disease process and caregiving among adults with cancer and family caregivers. Thereafter, through application of the BCW, seven intervention functions were selected: education, persuasion, incentivisation, environmental restructuring, training, modelling, and enablement.

The outcomes of previous work packages and the development of the intervention are described in more detail elsewhere $(19,20)$.

\section{Aims}

The proposed study aims to (1) test the feasibility of a socio-spiritual intervention suitable for the clinical realities of LMICs (primary outcome); and (2) evaluate the effectiveness of the socio-spiritual intervention on spiritual, family/social, and information needs, health literacy, and QoL (secondary outcome).

\section{Objectives}

The socio-spiritual intervention will be assessed according to the Donabedian Framework (23) of structure, process, and outcome as described below.

Structure: to evaluate rates of participant eligibility for inclusion, acceptance, and retention.

\section{Process:}

a. conduct the socio-spiritual intervention using an RCT design with adults with cancer and their family caregivers.

b. monitor intervention fidelity.

c. compare data collection rates for those allocated to the intervention and control groups.

Outcome: determine self-reported spiritual needs, family/social needs, information needs, cancer health literacy, and perceived QoL of adults with cancer and their family caregivers receiving the intervention (pre-post, intra and inter group comparison) compared to the control group.

\section{Hypotheses}

Adults with cancer and their family caregivers receiving the intervention will have significantly lower overall socio-spiritual needs, family/social needs, and information needs scores compared to the control 
group.

Adults with cancer and their family caregivers receiving the intervention will have significantly higher QoL and health literacy compared to the control group.

\section{Methods}

\section{Study design}

A single-centre, two-arm RCT will test the feasibility and evaluates efficacy of the socio-spiritual intervention for adults living with cancer and their family caregivers. Eligible participants will be randomly assigned to either the intervention or control group at a 1:1 ratio.

This study protocol was developed in accordance with the Standard Protocol Items: Recommendations for Interventional Trials (SPIRIT) statement guidelines for designing and reporting RCTs (24); the SPIRIT checklist (additional file 2) and the schedule of procedures can be seen in Table 1.

This trial was registered at the Pan African Clinical Trial Registry (www.pactr.org) (PACTR202007829295775) on 15 June 2020.

\section{Setting}

The study will be conducted at the Oncology Unit of a large, publicly funded tertiary referral hospital in Nigeria. Patients are referred to the hospital from all over the Northern region of Nigeria.

\section{Eligibility criteria}

Adults living with cancer and their family caregivers will be recruited. Eligibility criteria are adults (aged 18 or older) living with cancer (or adult family caregiver), receiving their first diagnosis within the last three months, literate to at least junior secondary school level (Grade 9) to complete self-administered questionnaires, and willingness to participate in a 2-hour program each week for four weeks. Exclusion criteria include patients with advanced cancer, or family caregivers who at the time of the study were also receiving treatment for cancer, or a condition that increased their physical and/or psychological vulnerability; unable to understand and speak English, and not able to complete self-reported surveys.

\section{Intervention}

The Behaviour Change Wheel framework was used to develop an evidence-based socio-spiritual intervention (referred to here as the "Zaman Lafiya Programme," a local vernacular name that means "Living Well"). Guided by the findings of a local needs survey, the intervention was designed to meet the needs of adult Nigerians living with cancer and their family caregivers to improve family/social support, spiritual support, and information/health literacy (20). The spiritual framework informed program content and processes to be congruent with the Nigerian culture. It was based on the assumption that lifestyles, social support, psychodynamics of ritual, psychodynamics of cognitive schemata, and supernatural 
effects influence QoL (see Figure 1). The intervention consists of four, two-hour sessions addressing different behavioural and social aspects that influence QoL. These factors encompass adult with cancerfamily caregiver dyadic (i) understanding cancer journey - need for family/social support and spirituality, (ii) sharing the journey - communication and building trusting relationships, (iii) finding the right support - family/social support and spiritual support and (iv) staying strong to overcome adversity understanding strengths and resources of the family by building spiritual support (see Table 2).

The intervention is a nurse-led, face-to-face program that includes activities and resources (video and paper) to facilitate intervention delivery. Group sessions will provide opportunities to express feelings and discuss issues of shared concern. For example, discussions will enable participants to gain an understanding of their experiences and how these may have changed their view of life, guiding them to adopt an optimistic cognitive framework for coping with stress and finding benefits in their situation.

A room at the hospital will be used for group sessions. The intervention will be delivered to adults with cancer-caregiver pairs (dyads) in a group format of five to seven dyads. Each group will run once a week for 4 weeks. Delivery of the program for all participants will be completed within four months. Researchers will contact participants by telephone in advance as a reminder about the forthcoming session and encourage attendance as much as possible. This approach aims to be supportive and identify any difficulties participants may be experiencing (such as transport; appointment clashes). All participants in the study will receive a nominal amount (\$10) for attendance which can be used to purchase a meal or pay for public transport.

\section{Control group}

Participants in the control arm will receive routine medical and nursing care which will also be provided to participants in the intervention arm. For ethical reasons, and if desired; participants assigned to the control group will be offered a printed copy of intervention resources and a one-day workshop after data collection is complete.

\section{Outcomes}

The primary and secondary outcomes are presented in Table 3. The study will follow the guidelines used by Leon., Davis. (25) to demonstrate feasibility of a study according to rates of eligibility (per cent screened for participants who were eligible), acceptance (proportion of those who screen eligible who enrol), retention (attrition and number of sessions completed by study arm), assessment process (proportion of planned assessments that are completed by participants), and intervention fidelity (percentage and similarity of session content covered across sessions by study facilitators).

Primary outcome measure - To test the feasibility (rates of eligibility, acceptance, retention, assessment process, and fidelity) of the intervention, research assistants will score:

Recruitment and acceptance rates at baseline (T1). 
Assessment process and fidelity will be throughout the trial.

Retention rate at $\mathrm{T} 2$.

Fidelity will be rated using a structured adherence checklist form to evaluate sessions in the study arm for a subset (50\%) session. The intervention fidelity scores range from 0 to 100 , depending on the number of topics discussed during the session.

Feasibility of the study will be demonstrated if $80 \%$ of participants complete all sessions and assessments. A $60 \%$ recruitment rate will be considered acceptable. The retention and assessment process benchmarks are $80 \%$ and covering 80 to $100 \%$ of topics would be regarded as high fidelity (26).

Secondary outcome measures - Efficacy of the intervention will include measures of the outcome variables (spiritual needs, family/social needs, information needs, health literacy, and QoL), repeated measurements will be conducted pre and post intervention. The surveys will be completed by participants in both the intervention and control groups.

\section{Participant's timeline}

For everyone in the intervention group, surveys will be completed at two-time points: baseline (T1), and immediately following completion of socio-spiritual intervention (four weeks, T2) (Figure 2) to determine whether the intervention has an immediate impact on the study outcomes. Participants in the control group will complete surveys within the same timeframe (baseline; + 4 weeks).

\section{Sample size}

A power analysis was performed to determine the sample size required to evaluate the efficacy of the intervention. Based on a previous related intervention study (27), a sample of 76 participants (38) dyads in each arm will provide $85 \%$ power for repeated-measures analysis of variance (time 1 vs time 2 ) with a moderate effect size and probability of 0.05. A final sample of 152 participants (adults with cancer and family caregivers) will be needed.

\section{Recruitment and consent}

Participants will be recruited at the oncology and outpatient clinics. All staff working in the oncology unit will be informed about the study by the research team. As shown in Figure 2, recruitment is a three-step process involving the following:

Initially identifying potential participants against inclusion and exclusion criteria: Nurses will identify and refer all potential participants who meet the inclusion criteria to research assistants who will be present at the clinic.

Approaching and informing potential participants of the study: The researcher will provide written and verbal information to adults living with cancer and family caregivers about the purpose and processes of 
the study. Patients who attend the clinic without a family caregiver but are interested in participating will be encouraged to discuss the study with their family, decide on a nominee, and notify the research assistant.

Consent: Those interested in the study will be given research information sheet, and then sign an informed consent form, which will be collected by the researcher.

\section{Randomisation and blinding}

Participants who meet the study criteria and give informed consent to participate in the study will be randomly assigned either to the intervention or control group (Figure 2). Computer-generated block randomisation will be performed to ensure equal (1:1) allocation. An independent researcher who is not involved in the study will carry out the allocation procedure. As a result, the research assistants who will be participating in group facilitation and participants will be blinded to the allocation of participants (allocation concealment). The allocation outcome will be communicated to research assistants by phone who will inform participants of their allocation and subsequent study procedure.

\section{Withdrawal criteria}

Participants are not under any obligation to participate in this trial. Therefore, they are also entirely free to discontinue participation at any time without penalty, or to decline to answer specific questions. The decision to participate in the study has no impact on the treatment participants receive from the Hospital.

\section{Data collection}

For all participants, socio-demographic data (age, gender, ethnicity, education, marital status, religion, economic status, relationship to the patient, duration of caregiving), clinical information (including cancer type, and stage of cancer), primary outcome (recruitment rate and acceptance, retention rate, and assessment process), and secondary outcomes (social/family needs, spiritual needs, information needs, health literacy, and QoL) will be measured. Well-validated measures will be used (see Table 3).

\section{Independent variables}

Social/family needs - The Multidimensional Scale of Perceived Social Support (MSPSS) is a brief instrument designed to measure perceptions of support from 3 sources: Family, Friends, and Significant Other. The scale is comprised of 12 items, with 4 items for each subscale (28). Each item is scored on a 7-point Likert scale from 1 = very strongly disagree to 7 = very strongly agree. The MSPSS was initially validated in western, high-income countries but has since been validated in a number of non-western settings and LMICs with good reliability ranging from 0.86 to $0.90(29,30)$. In this study, MSPSS will be used to measure both adults living with cancer and family caregivers' perception of the adequacy of the social support. 
Spiritual need - The Spiritual Needs Assessment for Patients (SNAP) comprises 23 items in three domains: psychosocial (5 items), spiritual (13 items), and religious (5 items) (31). Each question is scored on a 4-point Likert scale ranging from $1=$ very much to $4=$ not at all. The total score can vary from 23-92, and higher values reflect more spiritual needs (32). Good reliability ranging from $0.74-0.95$ has been reported (31).

A modified version of the SNAP will be used to measure the spiritual needs of the family caregiver. Three items will be modified ("getting in touch with other patients with similar illnesses" to "getting in touch with other family caregivers", "finding meaning in your experience of illness" to "finding meaning in your experience of caregiving", and "making decisions about your medical treatment that are in keeping with your spiritual or religious beliefs" to "making decisions about your loved one's medical treatment that are in keeping with their spiritual or religious beliefs") to reflect the family caregivers needs.

Information needs - The ten-item Information subscale of the Comprehensive Needs Assessment Tool for Cancer (CNAT) will be used to measure adults with cancer information needs (33). The eight-item Information subscale of the Comprehensive Needs Assessment Tool for Cancer- Caregivers (CNAT-C) (34) will measure family caregivers' information needs. Items are scored on a 4-point Likert scale ranging from $0=$ no need to $3=$ high need. The CNAT and CNAT-C have Cronbach's alpha internal consistency reliability ranging from of $0.79-0.97(33,34)$.

Cancer health literacy - Cancer health literacy (CHLT-6) (35) is a cancer-specific health literacy tool which differentiates between patients with adequate and limited cancer health literacy with a high degree of precision. Responses to the six items are scored as correct (1) or incorrect (0) and summed. Participants are considered to either have adequate cancer health literacy (total score 4 to 6 ) or limited cancer literacy (total score less than 4) (36). The CHLT-6 has a Cronbach's alpha internal consistency reliability of 0.96 0.99 (35). It has been tested and used in Nigeria with good reliability of 0.74 (20). In this study, CHLT-6 will be used to measure both adults living with cancer and family caregivers' health literacy.

\section{Dependent variable}

Quality of life - The Functional Assessment of Chronic Illness Therapy-Spirituality (FACIT-Sp) (version 4) combines the 27-item FACT-G and the 12-item FACIT-Sp $(37,38)$. FACIT-Sp will be used to assess the QoL of participants and a modified version will be administered to family caregivers. Items three, five, eleven, twelve, sixteen, seventeen, nineteen, twenty, twenty-four, thirty-eight, and thirty-nine were rephrased for family caregivers.

The survey consists of 39 items with five domains, assessing physical well-being (seven items), social/family well-being (seven items), emotional well-being (six items), functional well-being (seven items), and spiritual well-being (twelve items). Items are rated on a Likert-scale from 0 (not at all) to 4 (very much) (39). Higher scores indicate a more favourable perception of QoL (40). It has good internal consistency reliability ranging from 0.72 - 0.85 (41). 


\section{Data management and privacy procedures}

All data will be password protected. All identifying information of participants will be coded and deidentified. Signed informed consent forms and returned coded/anonymised surveys will be stored in a secured project file, and access will be restricted to research team members, who will all sign a privacy statement. Data will be entered into the Statistical Package for the Social Sciences (SPSS) version 27 (42) database by a researcher not involved with the intervention and blind to participants' allocation. Data integrity will be assured by random consistency checks/re-entry of data. Archived electronic data will be kept for a maximum of seven years. Collected data will be processed anonymously in publications and reports, preventing identification of individual participants.

\section{Approach to analysis}

Data will be entered, cleaned, and analysed using SPSS. All statistical analyses will be performed based on intention-to-treat.

Descriptive statistics - Continuous demographic and clinical variables will be summarised by means and standard deviations, while categorical data will be described using frequencies and percentages and $95 \%$ confidence intervals. Normality assumptions of continuous outcome variables will be evaluated.

Inferential statistics - Parametric or non-parametric inferential statistical tests will be performed to assess for differences between adults with cancer and family caregivers' demographic/medical variables (e.g., age, gender, economic status, cancer type, and dyadic relationship) in intervention and control groups at baseline. If differences are detected, these variables will be controlled in subsequent analyses. To assess intervention efficacy, repeated measures Multivariate Analysis of Variance (MANOVA) will be used to analyse changes in study variables from time 1 to 2 , with time and role (patient and caregiver) as within-subject variables to control for the correlated nature of the data.

\section{Validity and reliability}

Research assistant blinded to participant allocation and not involved in group facilitation will be responsible for collection of data. All instruments have been published and found to be reliable. The survey will be piloted with a Nigerian sample of five adults with cancer and five family caregivers. Group facilitators will have expertise in cancer care and group processes.

Steps would be taken to ensure intervention fidelity, such as ensuring that each participant received the same dose, and that the intervention period was set for a fixed number of groups. A programme manual was designed which contains precise detail of the content of each session. A programme manual will be developed that provides specific information about the content of each session.

Facilitators will undertake five days of training on the study aims, procedures, surveys, content of four sessions, group processes, use of registration forms, and data collection, during which they will become acquainted with the programme and their position within it. The importance of rigour will also be 
stressed. A digital version of the intervention protocol and facilitator guide will be made available to facilitators as part of their orientation and training for the study. The facilitators will be asked to fill out an intervention diary for each session in order to document any deviations from the programme. All analyses will be performed based on intention to treat.

\section{Ethical considerations}

The Declaration of Helsinki's ethical research principles will guide the study (43). The Ethics Committee of Ahmadu Bello University Teaching Hospital (ABUTH) Zaria, Nigeria (ABUTHZ/HREC/ W38/2020) and the Human Research Ethics Committee of Griffith University, Queensland, Australia (GU Ref No 2020/554) approved this study in May and July 2020, respectively. Written consent will be obtained from the participants.

Participation will be voluntary without coercion, manipulation, or undue influence. Participants will be duly informed that their consent is fluid and that they are free to withdraw at any point during the study process. The principle of justice and fairness will be adhered to by ensuring that all potential participants are treated equally without favour to individual on the grounds of religion, gender, age, or cadre.

\section{Dissemination policy}

After the study is completed, the findings will be disseminated across a variety of media, including openaccess peer-reviewed journals and national and international conferences. The demonstrated efficacy would improve the intervention's implementation in clinical practice and communities in low and middleincome countries. Furthermore, the findings will inform a larger longitudinal RCT study.

\section{Discussion}

This protocol describes an RCT to test the feasibility and efficacy of a nurse-delivered socio-spiritual intervention for adults living with cancer and their family caregivers in a LMICs. The primary focus will be on feasibility (recruitment rate, retention rate, and fidelity). Secondary outcomes evaluate the efficacy of this intervention in addressing spirituality, family/social needs, information needs and QoL among participants. The intervention is theory-driven and in response to a recent needs survey conducted in Nigeria for adults with cancer and their family caregivers (20). The emphasis on spirituality is novel and highly relevant to low-income countries such as Nigeria.

Research shows a dramatic rise in cancer incidence in many parts of Africa (44). Although incidence is lower than high-income countries, mortality rates in Africa are the highest in the world (45). The needs of patients with cancer and their family caregivers in low-income countries are greater than those in highincome countries, suggesting that African populations may share a higher burden of cancer. It is therefore imperative to develop and test brief, inexpensive, family-based interventions to assist coping and wellbeing. 
Most intervention studies in psycho-oncology research have been conducted in high-income countries (19). To the best of our knowledge, there has been no intervention research targeting socio-spiritual and information needs of the patient/caregiver dyad in Africa. With the rising prevalence of cancer, there is a great need for research on supportive therapy for individuals with cancer and family caregivers in Africa and Nigeria in particular.

In Nigeria, cancer is still seen as a spiritual issue with a superstitious belief in orthodox treatments, in addition to a family-centred model of decision-making that may influence how individuals access and respond to treatment. These are critical factors to be considered in resource-limited settings. The proposed intervention aims to address an often-neglected area of need. Results are expected to guide the further development and improvement of the intervention for future more extensive trials and application to other low and middle-income countries.

\section{Limitations}

This proposed study has some limitations, including being unfunded and conducted in a single tertiary centre. The heterogeneity of adults living with cancer with respect to types and stages of cancer would inevitably affect the psychosocial well-being of participants and influence various outcomes. Thus, variations in reported outcomes cannot be attributed solely to the proposed intervention. If confounding differences arise from demographic and medical variables, this will be controlled in the data analysis. Another limitation is that the intervention is limited to only those who speak English. This excludes nonEnglish - speaking adults with cancer and family caregivers who may have different needs. Generalizability of findings may therefore be limited to the English-speaking population.

\section{Conclusion}

This study describes a socio-spiritual intervention and proposed feasibility and evaluation for adults living with cancer and their family caregivers. The intervention was developed based on current literature, theoretical framework, and views of adults with cancer/family caregivers in Nigeria to maximise the efficacy and relevance of the target population. The intervention is important as there is currently an insufficient/poor provision of services for adults with cancer and their family caregivers in Nigeria. There is currently no provision in the form of interventions explicitly designed to meet the needs of this population in sub-Saharan Africa. Findings are expected to help assess and evaluate the likely success and efficacy of rolling out this intervention to more people in a range of settings.

\section{Trial status}

The trial is currently open for recruitment.

\section{Abbreviations}


ABUTH: Ahmadu Bello University Teaching Hospital; LMIC: Low-middle-income-countries; UK: United Kingdom; MANOVA: Multivariate Analysis of Variance; QoL: Quality of life; RCT: Randomised controlled trial; SPSS: Statistical Package for the Social Sciences; T1: Time 1; T2: Time 2; BCW: Behaviour Change Wheel; FACIT-Sp: Functional Assessment of Chronic Illness Therapy-Spirituality; FACT-G: Functional Assessment of Cancer Therapy - General; SNAP: Spiritual Needs Assessment for Patients; MSPSS: Multidimensional Scale of Perceived Social Support; Subscale of the Comprehensive Needs Assessment Tool for Cancer; CNAT: Comprehensive Needs Assessment Tool for Cancer; CNAT-C: Comprehensive Needs Assessment Tool for Cancer- Caregivers; CHLT-6: Cancer health literacy- 6 items; SPIRIT: Standard Protocol Items: Recommendations for Interventional Trials.

\section{Declarations}

Ethics approval and consent to participate - The Ethics Committee of Ahmadu Bello University Teaching Hospital (ABUTH) Zaria, Nigeria (ABUTHZ/HREC/ W38/2020) and the Human Research Ethics Committee of Griffith University, Queensland, Australia (GU Ref No 2020/554) approved this study in May and July 2020 , respectively. Written consent will be obtained from the participants.

Consent for publication - Not applicable.

Availability of data and materials - After the trial is completed, the corresponding author will make the final data generated and/or analysed available upon reasonable request. Access to the final datasets will be in accordance with governance policies.

Competing interests - The authors declare that they have no competing interests.

Funding - The first author is supported by a Griffith University International Postgraduate Research Scholarship.

\section{Authors' contributions -}

Study design: I.G., D.K.C., and E.C.

Manuscript writing and revisions for important intellectual content: I.G., D.K.C., and E.C.

All authors read and approved the final manuscript.

Acknowledgements - Not applicable.

\section{References}

1. Lekoane KMB, Kuupiel D, Mashamba-Thompson TP, Ginindza TG. Evidence on the prevalence, incidence, mortality and trends of human papilloma virus-associated cancers in sub-Saharan Africa: Systematic scoping review. BMC Cancer. 2019;19(1):563-10. 
2. Pilleron S, Soerjomataram I, Charvat $\mathrm{H}$, Chokunonga E, Somdyala NIM, Wabinga $\mathrm{H}$, et al. Cancer incidence in older adults in selected regions of sub-Saharan Africa, 2008-2012. International journal of cancer. 2019;144(8):1824-33.

3. Boyle P, Ngoma T, Sullivan R, Brawley O. Cancer in Africa: the way forward. ecancermedicalscience. 2019;13.

4. Shah K, Peek H. Cancer Control in Low- and Middle-Income Countries: Is It Time to Consider Screening? Journal of global oncology. 2019;5(5):1-8.

5. Ferrell BR, Kravitz K, Borneman T, Friedmann ET. Family caregivers: A qualitative study to better understand the quality-of-life concerns and needs of this population. Clin J Oncol Nurs. 2018;22(3):286-94.

6. Gabriel IO, Mayers PM. Effects of a psychosocial intervention on the quality of life of primary caregivers of women with breast cancer. European Journal of Oncology Nursing. 2019;38:85-91.

7. Chae BJ, Lee J, Lee SK, Shin H-J, Jung S-Y, Lee JW, et al. Unmet needs and related factors of Korean breast cancer survivors: a multicenter, cross-sectional study. BMC Cancer. 2019;19(1):839-16.

8. Akuoko CP, Armah E, Sarpong T, Quansah DY, Amankwaa I, Boateng D. Barriers to early presentation and diagnosis of breast cancer among African women living in sub-Saharan Africa. PloS one. 2017;12(2):e0171024.

9. Tetteh D, Faulkner S. Sociocultural factors and breast cancer in sub-Saharan Africa: implications for diagnosis and management. Women's Health. 2016;12(1):147-56.

10. Sarki A, Roni B. This disease is "not for hospital": myths and misconceptions about cancers in Northern Nigeria. Health Rep. 2019;3:e2019070.

11. Shin DW, Park HS, Lee SH, Jeon SH, Cho S, Kang SH, et al. Health-related quality of life, perceived social support, and depression in disease-free survivors who underwent curative surgery only for prostate, kidney and bladder cancer: Comparison among survivors and with the general population. Cancer research treatment: official journal of Korean Cancer Association. 2019;51(1):289.

12. Santin O, Murray L, Prue G, Gavin A, Gormley G, Donnelly M. Self-reported psychosocial needs and health-related quality of life of colorectal cancer survivors. European Journal of Oncology Nursing. 2015;19(4):336-42.

13. Park B-W, Hwang SY. Unmet Needs and Their Relationship with Quality of Life among Women with Recurrent Breast Cancer. Journal of Breast Cancer. 2012;15(4):454-61.

14. Teo I, Krishnan A, Lee GL. Psychosocial interventions for advanced cancer patients: A systematic review. Psycho-Oncology. 2019;28(7):1394-407.

15. Son H, Son Y-J, Kim H, Lee Y. Effect of psychosocial interventions on the quality of life of patients with colorectal cancer: A systematic review and meta-analysis. Health Quality of Life Outcomes. 2018;16(1):119-.

16. Badger TA, Segrin C, Sikorskii A, Pasvogel A, Weihs K, Lopez AM, et al. Randomized controlled trial of supportive care interventions to manage psychological distress and symptoms in Latinas with breast cancer and their informal caregivers. Psychology \& health. 2019:1-20. 
17. Badr H, Carmack CL, Diefenbach MA. Psychosocial Interventions for Patients and Caregivers in the Age of New Communication Technologies: Opportunities and Challenges in Cancer Care. Journal of Health Communication. 2015;20(3):328-42.

18. Tan JYS, Lam KFY, Lim HA, Chua SM, Kua EH, Griva K, et al. Post-intervention sustainability of a brief psycho-educational support group intervention for family caregivers of cancer patients. Asia-Pacific Psychiatry. 2018;10(3):e12305. -n/a.

19. Gabriel I, Creedy D, Coyne E. A systematic review of psychosocial interventions to improve quality of life of people with cancer and their family caregivers. Nursing open. 2020.

20. Gabriel I, Creedy D, Coyne E. Quality of life and associated factors among adults living with cancer and their family caregivers. Nursing \& health sciences; 2021.

21. Hodge DR. Spirituality. Towards a theoretical framework. Social Thought. 2000;19(4):1-20.

22. Michie S, Van Stralen MM, West R. The behaviour change wheel: a new method for characterising and designing behaviour change interventions. Implementation science. 2011;6(1):1-12.

23. Donabedian. The quality of medical care. Methods for assessing and monitoring the quality of care for research and for quality assurance programs. Science. 1978;200(4344):856-64.

24. Chan A-W, Tetzlaff JM, Altman DG, Laupacis A, Gøtzsche PC, Krleža-Jerić K, et al. SPIRIT 2013 statement: defining standard protocol items for clinical trials. Ann Intern Med. 2013;158(3):200-7.

25. Leon D. Kraemer. The role and interpretation of pilot studies in clinical research. J Psychiatr Res. 2011;45(5):626-9.

26. Borrelli B. The assessment, monitoring, and enhancement of treatment fidelity in public health clinical trials. J Public Health Dent. 2011;71:52-63.

27. Northouse L, Schafenacker A, Barr KLC, Katapodi M, Yoon H, Brittain K, et al. A Tailored Web-Based Psychoeducational Intervention for Cancer Patients and Their Family Caregivers. Cancer Nurs. 2014;37(5):321-30.

28. Zimet GD, Dahlem NW, Zimet SG, Farley GK. The multidimensional scale of perceived social support. J Pers Assess. 1988;52(1):30-41.

29. Bruwer B, Emsley R, Kidd M, Lochner C, Seedat S. Psychometric properties of the Multidimensional Scale of Perceived Social Support in youth. Compr Psychiatr. 2008;49(2):195-201.

30. Qadir F, Khalid A, Haqqani S, Medhin G. The association of marital relationship and perceived social support with mental health of women in Pakistan. BMC Public Health. 2013;13(1):1-13.

31. Sharma RKMDMHS, Astrow ABMD, Texeira KP, Sulmasy DPMDP. The Spiritual Needs Assessment for Patients (SNAP): Development and Validation of a Comprehensive Instrument to Assess Unmet Spiritual Needs. J Pain Symptom Manag. 2012;44(1):44-51.

32. De Araujo Toloi D, Uema D, Matsushita F, Da Silva Andrade PA, Branco TP, De Carvalho Chino FTB, et al. Validation of questionnaire on the Spiritual Needs Assessment for Patients (SNAP) questionnaire in Brazilian Portuguese. Ecancermedicalscience. 2016;10:694- 
33. Shim E-J, Lee k-S, Park J-H, Park J-H. Comprehensive needs assessment tool in cancer (CNAT): the development and validation. Support Care Cancer. 2011;19(12):1957-68.

34. Shin DW, Park JH, Shim EJ, Park JH, Choi JY, Kim SG, et al. The development of a comprehensive needs assessment tool for cancer-caregivers in patient-caregiver dyads. Psycho-Oncology. 2011;20(12):1342-52.

35. Dumenci L, Matsuyama R, Riddle DL, Cartwright LA, Perera RA, Chung H, et al. Measurement of Cancer Health Literacy and Identification of Patients with Limited Cancer Health Literacy. Journal of Health Communication. 2014;19(sup2):205-24.

36. Kanu $\mathrm{CN}$. The relationship between health literacy, patient activation, and health outcomes in breast cancer patients 2019.

37. Canada AL, Murphy PE, Fitchett G, Peterman AH, Schover LR. A 3-factor model for the FACIT-Sp. Psycho-oncology. 2008;17(9):908-16.

38. Cella DF, Tulsky DS, Gray G, Sarafian B, Linn E, Bonomi A, et al. The Functional Assessment of Cancer Therapy scale: development and validation of the general measure. J Clin Oncol. 1993;11(3):570-9.

39. Schubart JRPD, Wise JDM, Deshaies IMD, Kimchi ETMD, Staveley-O'Carroll KFMDPD, Gusani NJMD. Quality of Life Assessment in Postoperative Patients with Upper GI Malignancies. J Surg Res. 2010;163(1):40-6.

40. Weisbord SD, Carmody SS, Bruns FJ, Rotondi AJ, Cohen LM, Zeidel ML, et al. Symptom burden, quality of life, advance care planning and the potential value of palliative care in severely ill haemodialysis patients. Nephrology Dialysis Transplantation. 2003;18(7):1345-52.

41. Peterman AH, Fitchett G, Brady MJ, Hernandez L, Cella D. Measuring spiritual well-being in people with cancer: the functional assessment of chronic illness therapy-Spiritual Well-being Scale (FACIT$\mathrm{Sp})$. Annals of behavioral medicine. 2002;24(1):49-58.

42. George D, Mallery P. IBM SPSS statistics 26 step by step: A simple guide and reference. Routledge; 2019.

43. World Medical Association Declaration. of Helsinki: ethical principles for medical research involving human subjects. Jama. 2013;310(20):2191-4.

44. Ferlay J, Soerjomataram I, Dikshit R, Eser S, Mathers C, Rebelo M, et al. Cancer incidence and mortality worldwide: sources, methods and major patterns in GLOBOCAN 2012. International journal of cancer. 2015;136(5):E359-E86.

45. Azubuike SO, Muirhead C, Hayes L, McNally R. Rising global burden of breast cancer: The case of sub-Saharan Africa (with emphasis on Nigeria) and implications for regional development: A review. World Journal of Surgical Oncology. 2018;16(1):63-13.

\section{Tables}

Table 1: SPIRIT protocol schedule of procedures 


\begin{tabular}{|c|c|c|c|c|c|}
\hline & & & JDY PERIC & & \\
\hline & $\begin{array}{c}\text { Pre- } \\
\text { allocation }\end{array}$ & Randomisation & $\begin{array}{c}\text { Basement } \\
\text { Assessment }\end{array}$ & $\begin{array}{c}\text { Intervention } \\
\text { Period }\end{array}$ & $\begin{array}{c}\text { 4-week } \\
\text { follow up }\end{array}$ \\
\hline TIME POINT & 0 & $\mathbf{0}$ & Tl & 0 & T2 \\
\hline ENROLMENT: & & & & & \\
\hline Eligibility screen & $\mathrm{X}$ & & & & \\
\hline & $\mathrm{X}$ & & & & \\
\hline & $\mathrm{X}$ & & & & \\
\hline ( & & $\mathrm{x}$ & & & \\
\hline Baseline survey & & & $\mathrm{x}$ & & \\
\hline INTERVENTION: & & & & & \\
\hline $\begin{array}{l}\text { Intervention group: Zaman Lafiya } \\
\text { Programme and Routine care }\end{array}$ & & & & $\mathrm{x}$ & \\
\hline Control group: Routine care & & & & & \\
\hline ASSESSMENTS: & & & & & \\
\hline & & & & $\mathrm{x}$ & \\
\hline & & & & $\mathrm{X}$ & \\
\hline & & & & $\mathrm{X}$ & \\
\hline & & & & $\mathrm{X}$ & \\
\hline Training delivery staff log & & & & & \\
\hline & & & & & $\mathrm{X}$ \\
\hline
\end{tabular}

Table 2: Socio-spiritual intervention content 
$\begin{array}{ll}\text { Theme } & \begin{array}{l}\text { Typical adult with cancer/ caregivers' } \\ \text { concerns }\end{array}\end{array}$

Understanding

cancer journey -

need for

family/social

support and

spirituality.

- Assess baseline health literacy of the disease. Discuss results amongst the group.

- Educate about disease and treatments according to identified knowledge gaps and misconceptions.

- Discuss communication strategies and how to be assertive to obtain additional information

- Clearly and concisely communicate and demonstrate a range of possible therapies such as relaxation and massage.

\section{The spiritual Framework's discrete pathways}

Intervention

- Health
promotive
behaviours and
lifestyles.
- Social support.
-
Psychodynamics
of ritual.
-
Psychodynamics
of cognitive
schemata,
illness and caregiving according to identified
- Assess baseline health literacy of the disease and caregiving. Discuss results amongst the group.

- Educate about treatments and knowledge gaps and misconceptions.

- Discuss communication strategies and how to be assertive to obtain additional information.

- Clearly and concisely communicate and demonstrate a range of possible therapies such as relaxation, massage.

- Ask participants to write down their concerns and fears. Discuss results amongst the group.

- Discuss strategies to promote open communication. 
- Brainstorm strategies to enhance mutual support and teamwork.

- Highlight the value of living "here and now" and promote approaches to helping dyad work within the limits of their new limitations.

Finding the right Loneliness and reduced social support - family/ support.

social support and spiritual support.
Stay strong overcoming adversity understanding strengths and resources of the family building spiritual support.

\section{- Social support. Psychodynamics of ritual. \\ Psychodynamics of cognitive schemata.}

Coming to terms with "who I am now." Creating meaning, finding purpose; achieve a sense of wisdom through life's experience and lessons.
- Health

promotive behaviours and lifestyles.

- Social support.

Psychodynamics
- Identify family strengths using a short survey. Discuss results amongst the group.

- Social connections; link between the past and present.

- Provide realityoriented approaches and pragmatic social support maintain regular and brief interventions.

- Work to address cognitive distortions and devastating behaviours.
- Use reflective writing to deepen insights, reflect on life changes and what is essential in life. 
of ritual.

-

Psychodynamics

of cognitive

schemata.

- Supernatural effects.
- Discuss the use of spiritual coping with health challenges, especially dealing with those situations that are not in our control.

- Consider what provides a sense of inner peace for the individual.

- Explore the views of each other and come to a new shared understanding.

\section{Table 3: Overview of study outcomes}




\begin{tabular}{|c|c|c|c|c|c|c|c|}
\hline \multirow[t]{2}{*}{ Outcome } & \multirow{2}{*}{$\begin{array}{l}\text { Domain } \\
\text { (Aim) }\end{array}$} & \multirow[t]{2}{*}{ Name } & \multirow[t]{2}{*}{ Source } & \multicolumn{4}{|c|}{ Time point } \\
\hline & & & & $\begin{array}{l}\text { W1 } \\
\text { (T1) }\end{array}$ & W2 & W3 & $\begin{array}{l}\text { W4 } \\
\text { (T2) }\end{array}$ \\
\hline Primary & $\begin{array}{l}\text { Feasibility } \\
\text { (1) }\end{array}$ & $\begin{array}{l}\text { Recruitment } \\
\text { rate }\end{array}$ & Study records & $x$ & & & \\
\hline Primary & $\begin{array}{l}\text { Feasibility } \\
\text { (1) }\end{array}$ & Acceptance & Study records & $x$ & & & \\
\hline Primary & $\begin{array}{l}\text { Feasibility } \\
\text { (1) }\end{array}$ & $\begin{array}{l}\text { Retention } \\
\text { rate }\end{array}$ & Study records & & & & $\mathrm{x}$ \\
\hline Primary & $\begin{array}{l}\text { Feasibility } \\
\text { (1) }\end{array}$ & $\begin{array}{l}\text { Assessment } \\
\text { process }\end{array}$ & Study records & $x$ & $x$ & $x$ & $\mathrm{x}$ \\
\hline Primary & $\begin{array}{l}\text { Feasibility } \\
\text { (1) }\end{array}$ & Fidelity & $\begin{array}{l}\text { Fidelity rating form - done } \\
\text { at every session. }\end{array}$ & $x$ & $x$ & $x$ & $x$ \\
\hline \multirow[t]{2}{*}{ Secondary } & $\begin{array}{l}\text { Preliminary } \\
\text { efficacy ( } 2)\end{array}$ & $\begin{array}{l}\text { Spiritual } \\
\text { needs }\end{array}$ & $\begin{array}{l}\text { - The Spiritual Needs } \\
\text { Assessment for Patients } \\
\text { (SNAP) (Likert scale } 23 \\
\text { items) }\end{array}$ & $\mathrm{x}$ & & & $\mathrm{x}$ \\
\hline & & & $\begin{array}{l}\text { - Modified version of the } \\
\text { Spiritual Needs } \\
\text { Assessment for Patient } \\
\text { (SNAP) (Likert scale } 23 \\
\text { items) }\end{array}$ & & & & \\
\hline Secondary & $\begin{array}{l}\text { Preliminary } \\
\text { efficacy (2) }\end{array}$ & Social needs & $\begin{array}{l}\text { - Multidimensional Scale } \\
\text { of Perceived Social } \\
\text { Support (MSPSS) (Survey: } \\
\text { Likert scale } 12 \text { items) }\end{array}$ & $\mathrm{x}$ & & & $\mathrm{x}$ \\
\hline \multirow[t]{2}{*}{ Secondary } & $\begin{array}{l}\text { Preliminary } \\
\text { efficacy ( } 2 \text { ) }\end{array}$ & $\begin{array}{l}\text { Information } \\
\text { need }\end{array}$ & $\begin{array}{l}\text { - Information Scale of the } \\
\text { Comprehensive Needs } \\
\text { Assessment Tool for } \\
\text { Cancer (Likert scale } 10 \\
\text { items) and Cancer health } \\
\text { literacy (CHLT-6) }\end{array}$ & $\mathrm{x}$ & & & $\mathrm{x}$ \\
\hline & & & $\begin{array}{l}\text { - Information Scale of the } \\
\text { Comprehensive Needs } \\
\text { Assessment Tool for } \\
\text { Cancer- Caregivers (Likert } \\
\text { scale } 8 \text { items) and Cancer } \\
\text { health literacy (CHLT-6) }\end{array}$ & & & & \\
\hline
\end{tabular}

Page 21/23 


\section{Secondary}

Preliminary Quality of efficacy (2) life
- The Functional

Assessment of Chronic

Illness Therapy-Spirituality

(FACIT-Sp) (Likert scale 39

items)

- The Functional

Assessment of Chronic

Illness Therapy-Spirituality

(FACIT-Sp) (Likert scale 39

items) (Modified)

\section{Descriptive \\ (2) \\ Demographic and clinical information \\ Socio-demographic and health-related information (10 items for adults with cancer and 9 items for family caregivers).}

Key: W: Week; T1: Time 1; T2: Time 2.

\section{Figures}

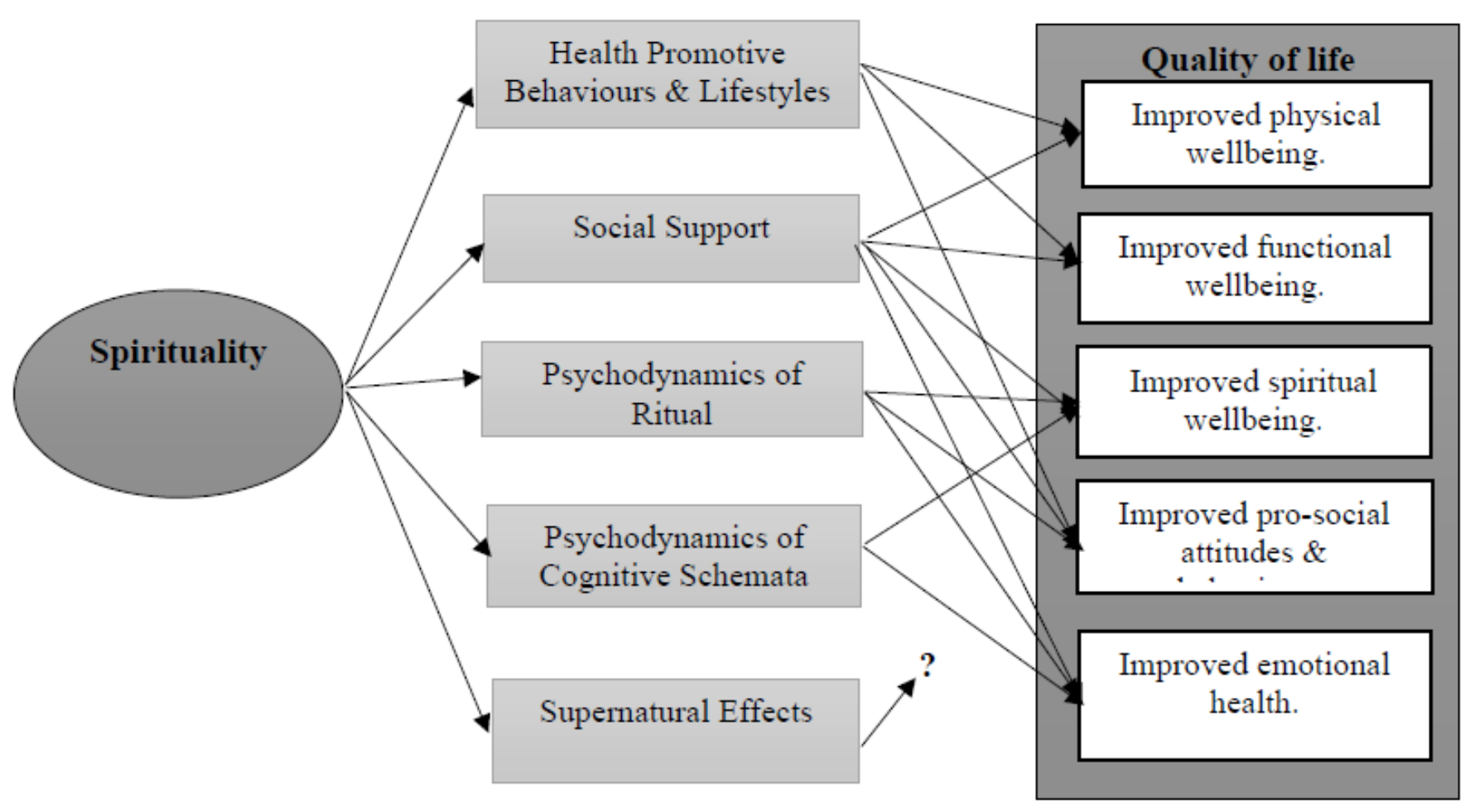

Figure 1 


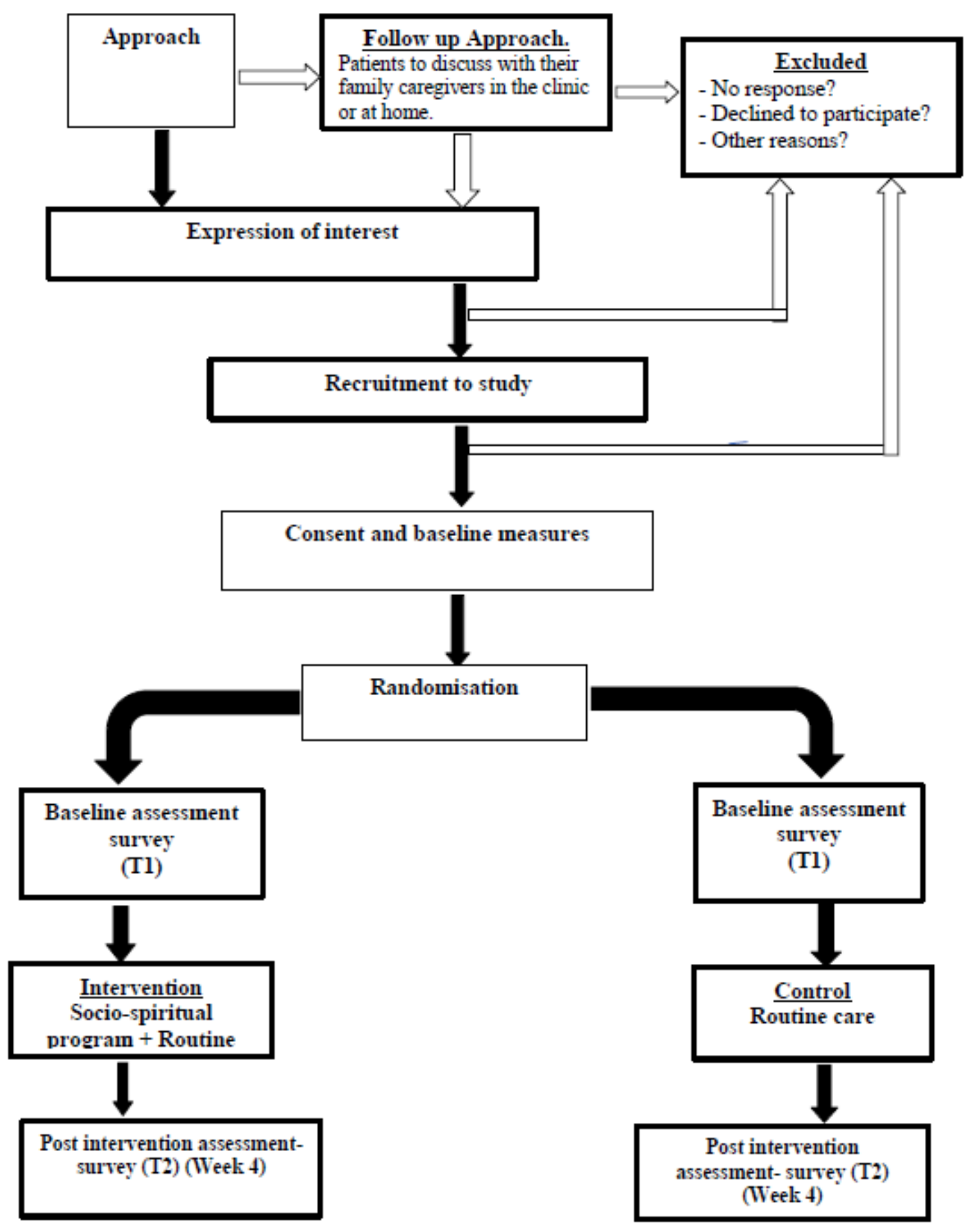

Figure 2

Participant flow chart

\section{Supplementary Files}

This is a list of supplementary files associated with this preprint. Click to download.

- SPIRITChecklistFILE2.pdf 